\title{
Are novel non-invasive imaging techniques needed in patients with suspected prosthetic heart valve endocarditis? A systematic review and meta-analysis
}

\author{
Jesse Habets • Wilco Tanis • Johannes B. Reitsma • \\ Renee B. A. van den Brink • Willem P. Th. M. Mali • \\ Steven A. J. Chamuleau • Ricardo P. J. Budde
}

Received: 26 June 2014 / Revised: 10 November 2014 / Accepted: 15 January 2015 / Published online: 14 February 2015

(C) The Author(s) 2015. This article is published with open access at Springerlink.com

\begin{abstract}
Objectives Multimodal non-invasive imaging plays a key role in establishing a diagnosis of PHV endocarditis. The objective of this study was to provide a systematic review of the literature and meta-analysis of the diagnostic accuracy of TTE, TEE, and MDCT in patients with (suspected) PHV endocarditis.

Methods Studies published between 1985 and 2013 were identified via search and cross-reference of PubMed/Embase databases. Studies were included if (1) they reported on the
\end{abstract}

Jesse Habets and Wilco Tanis contributed equally to this work.

Electronic supplementary material The online version of this article (doi:10.1007/s00330-015-3605-7) contains supplementary material, which is available to authorized users.

J. Habets $(\triangle) \cdot$ W. P. T. M. Mali

Department of Radiology, University Medical Center Utrecht, Heidelberglaan 100, 3508 GA Utrecht, The Netherlands

e-mail: J.Habets@umcutrecht.nl

W. Tanis

Department of Cardiology, Haga Teaching Hospital, The Hague, The Netherlands

J. B. Reitsma

Julius Center for Health Sciences and Primary Care, University

Medical Center Utrecht, Utrecht, The Netherlands

R. B. A. van den Brink

Department of Cardiology, Academic Medical Center,

Amsterdam, The Netherlands

S. A. J. Chamuleau

Department of Cardiology, University Medical Center Utrecht,

Utrecht, The Netherlands

R. P. J. Budde

Department of Radiology, Erasmus Medical Center, Rotterdam, The Netherlands non-invasive index tests TTE, TEE, or MDCT; (2) data was provided on PHV endocarditis as the condition of interest; and (3) imaging results were verified against either surgical inspection/autopsy or clinical follow-up reference standards, thereby enabling the extraction of 2-by-2 tables.

Results Twenty articles (including 496 patients) met the inclusion criteria for PHV endocarditis. TTE, TEE, and MDCT+ TEE had a pooled sensitivity/specificity for vegetations of 29/ $100 \%$; 82/95 \%, and 88/94\%, respectively. The pooled sensitivity/specificity of TTE, TEE, and MDCT+TEE for periannular complications was 36/93 \%, 86/98 \%, and $100 /$ $94 \%$, respectively.

Conclusions TEE showed good sensitivity and specificity for establishing a diagnosis of PHV endocarditis. Although MDCT data are limited, this review showed that MDCT in addition to TEE may improve sensitivity in detecting lifethreatening periannular complications.

Key Points

- Multimodal imaging is an important ingredient of diagnostic workup for PHV endocarditis.

- Transthoracic and transesophageal echography may miss life-threatening periannular complications.

- MDCT can improve sensitivity for the detection of lifethreatening periannular complications.

Keywords Echocardiography · Computed tomography . Endocarditis $\cdot$ Prosthetic heart valve $\cdot$ Systematic review

\section{Introduction}

Left-sided native heart valve disease often requires prosthetic heart valve (PHV) implantation, especially in the aortic position. In 2003, approximately 290,000 patients worldwide 
underwent heart valve replacement, and this figure is expected to rise, largely as a result of the ageing population, reaching an annual incidence of 850,000 by 2050 [1]. Although PHV implantation is a lifesaving surgical procedure, its major drawback is the risk of complications such as PHV obstruction or PHV endocarditis. PHV endocarditis is a life-threatening disease, with an incidence of $0.3-1.2 \%$ per patient-year, and is associated with high rates of mortality and comorbidity as well as substantial health care costs [2].

In clinical practice, PHV endocarditis presents with a heterogeneous spectrum of clinical manifestations such as fever, heart failure symptoms, or systematic embolization. According to the modified Duke criteria, echocardiography plays a pivotal role in establishing a diagnosis of PHV endocarditis [3]. TTE is the first-line clinical screening tool for the detection of PHV endocarditis, but often fails to detect positive signs of the disease such as vegetations and periannular complications (abscesses/mycotic aneurysms). TEE can add incremental value to TTE, but may still fail to detect life-threatening signs of PHV endocarditis. Multidetector-row computed tomography (MDCT) has recently emerged as a promising novel imaging technique for evaluating PHVs, and may provide complementary diagnostic information to echocardiography in patients with suspected PHV endocarditis [4].

The purpose of this systematic review was to compare the diagnostic accuracy of transthoracic echocardiography (TTE), transoesophageal echocardiography (TEE), and multidetector-row computed tomography (MDCT) plus TEE in patients with (suspected) PHV endocarditis.

\section{Material and methods}

\section{Literature search}

A systematic electronic search was performed in the PubMed and Embase databases for original works published through July 23, 2013. Articles were restricted to English language publications, and studies published before 1985 were excluded. Key search terms included the non-invasive imaging modalities TTE, TEE, and MDCT, and prosthetic heart valves and corresponding synonyms (Appendix I). Crossreferencing was performed for all full-text papers included.

\section{Selection of publications}

After duplicates were removed, the titles and abstracts were independently screened by two reviewers (JH and WT). Articles were included if they met three inclusion criteria. 1) The studies reported on one of the following non-invasive index tests: transthoracic echocardiogram (TTE), transesophageal echocardiogram (TEE), or retrospectively ECG-gated or prospectively ECG-triggered multidetector-row computed tomography (MDCT). 2) They provided data on PHV endocarditis as the condition of interest. 3) Imaging results were verified against the reference standard of surgical inspection/ autopsy or clinical follow-up, thereby enabling the extraction of 2-by-2 tables. Full-text publications of the included articles were obtained and were assessed independently by the two reviewers, and publications and data extraction were then extensively discussed in a consensus meeting.

\section{Quality assessment}

Information was collected on patient populations, study enrolment, non-invasive imaging modalities, and reference standard. Studies were systematically assessed for quality based on the validated Quality Assessment of Diagnostic Accuracy Studies (QUADAS)-2 checklist [5], which assesses the risk of bias and clinical applicability of studies based on four key domains: patient selection, index test, reference standard, and flow and timing.

\section{Data analysis}

The diagnostic accuracy of the different imaging modalities was assessed for the detection of the following signs of PHV endocarditis: vegetations, periannular complications (abscesses, mycotic aneurysms/pseudoaneurysms), PHV dehiscence, and PHV endocarditis in general. These signs of interest were defined according to echocardiographic criteria as follows: 1) vegetations, defined as irregularly shaped oscillating masses adherent to and distinct from the myocardium; 2) abscesses, defined as irregularly shaped inhomogeneous paravalvular enclosed masses within the periannular region, myocardium, or pericardium; 3) mycotic aneurysms/pseudoaneurysms, defined as echo-free perivalvular cavities with flow communicating with the cardiovascular lumen; and 4) PHV dehiscence, defined as a rocking motion of a PHV [2, 6]. PHV endocarditis in general included one or more of the above-mentioned signs of PHV endocarditis.

Forest plots of sensitivity and specificity were generated, along with their corresponding $95 \%$ confidence intervals (CI), and were stratified by target conditions and data within each forest plot grouped by index test (TTE, TEE, or MDCT+ TEE). The bivariate random effects model was used to compare summary estimates of sensitivity and specificity for each of the index tests. The bivariate approach simultaneously models pairs of (logit-transformed) sensitivity and specificity from studies, thereby incorporating any correlation that might exist between sensitivity and specificity. The model uses a random effects approach for both sensitivity and specificity, allowing for heterogeneity beyond chance due to clinical and methodological differences among studies. In the event that the results displayed no variation beyond that expected by 
chance, models were simplified to fixed-effects pooling of sensitivity, specificity, or both. To compare index tests, we extended the bivariate model with a covariate indicating the type of index test. Such a model calculates different summary estimates for sensitivity and specificity for each index test and also provides a formal test to determine whether differences are statistically different. The non-linear mixed models procedure (PROC NLMIXED) in SAS 9.2 (SAS Institute, Cary, NC, USA) was used to estimate the parameters of the bivariate models. $P$ values less than 0.05 were considered statistically significant.

\section{Results}

\section{Search results}

The systematic electronic search yielded a total of 89 publications after screening of titles and abstracts. Sixty full-text versions of the studies were obtained, and 45 studies were excluded for various reasons (Fig. 1). Cross-referencing of all included full-text articles resulted in six additional articles, and our recently accepted paper was added for completeness [7]. The final selection of articles included 22 studies for systematic review. Two studies reporting on PHV endocarditis were excluded because no diagnostic accuracy data were available $[8,9]$; therefore, 20 studies were ultimately included in the meta-analysis.

PHV endocarditis

Twenty-two studies reporting on the echocardiographic (TTE/ TEE) detection of signs of endocarditis using were included in the systematic review (Table 1) [7-27]. Three studies reported on retrospectively ECG-gated MDCT findings [7, 10, 28]. Data were prospectively (dedicated data collection; $n=11$; $50 \%$ ) and retrospectively (routine care data; $n=11 ; 50 \%$ ) collected (Table 1). In 15 (68\%) studies, the inclusion period occurred completely or partially in 1990 or earlier. The reference standard was exclusively surgical inspection or autopsy
Fig. 1 Systematic literature search

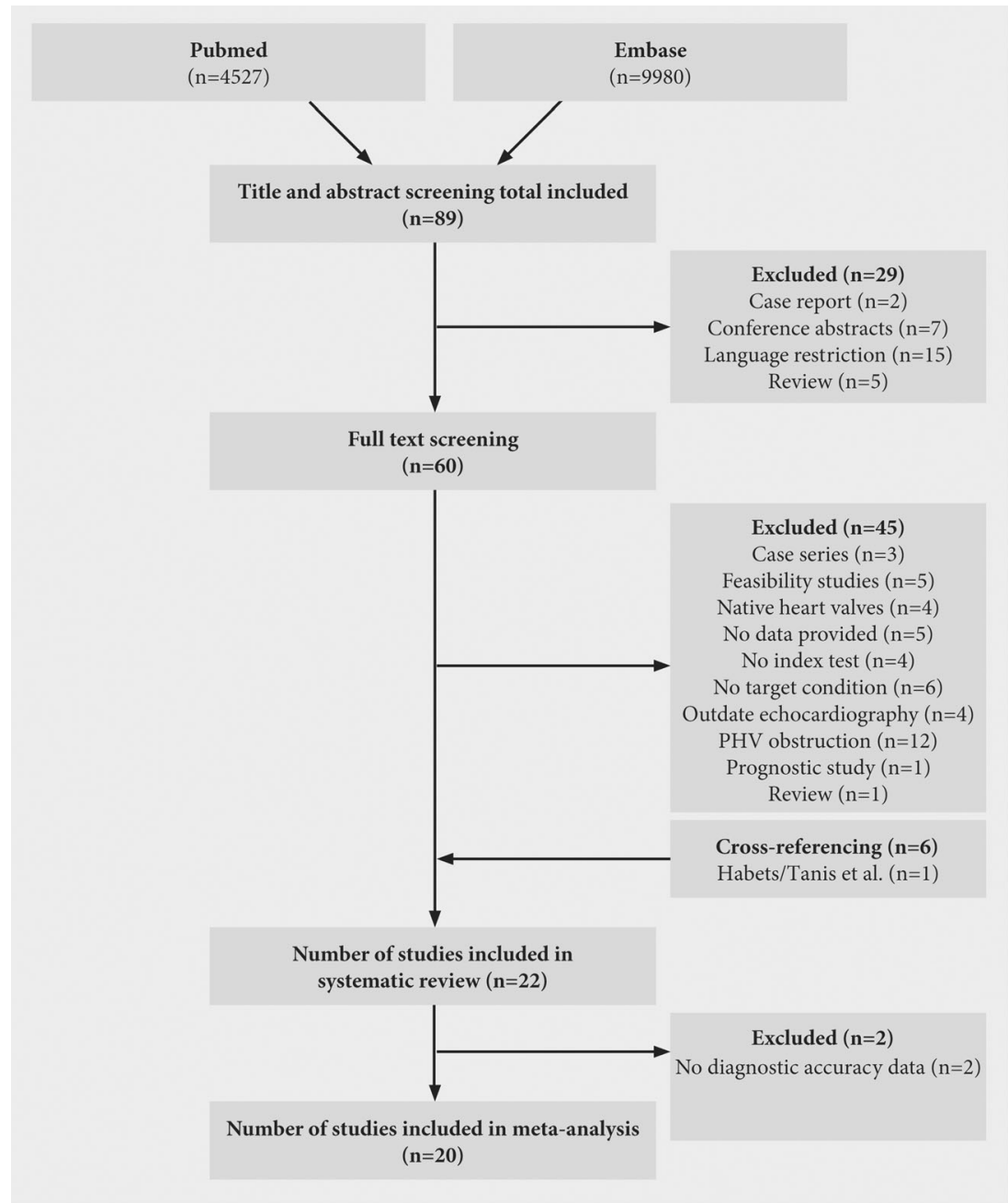




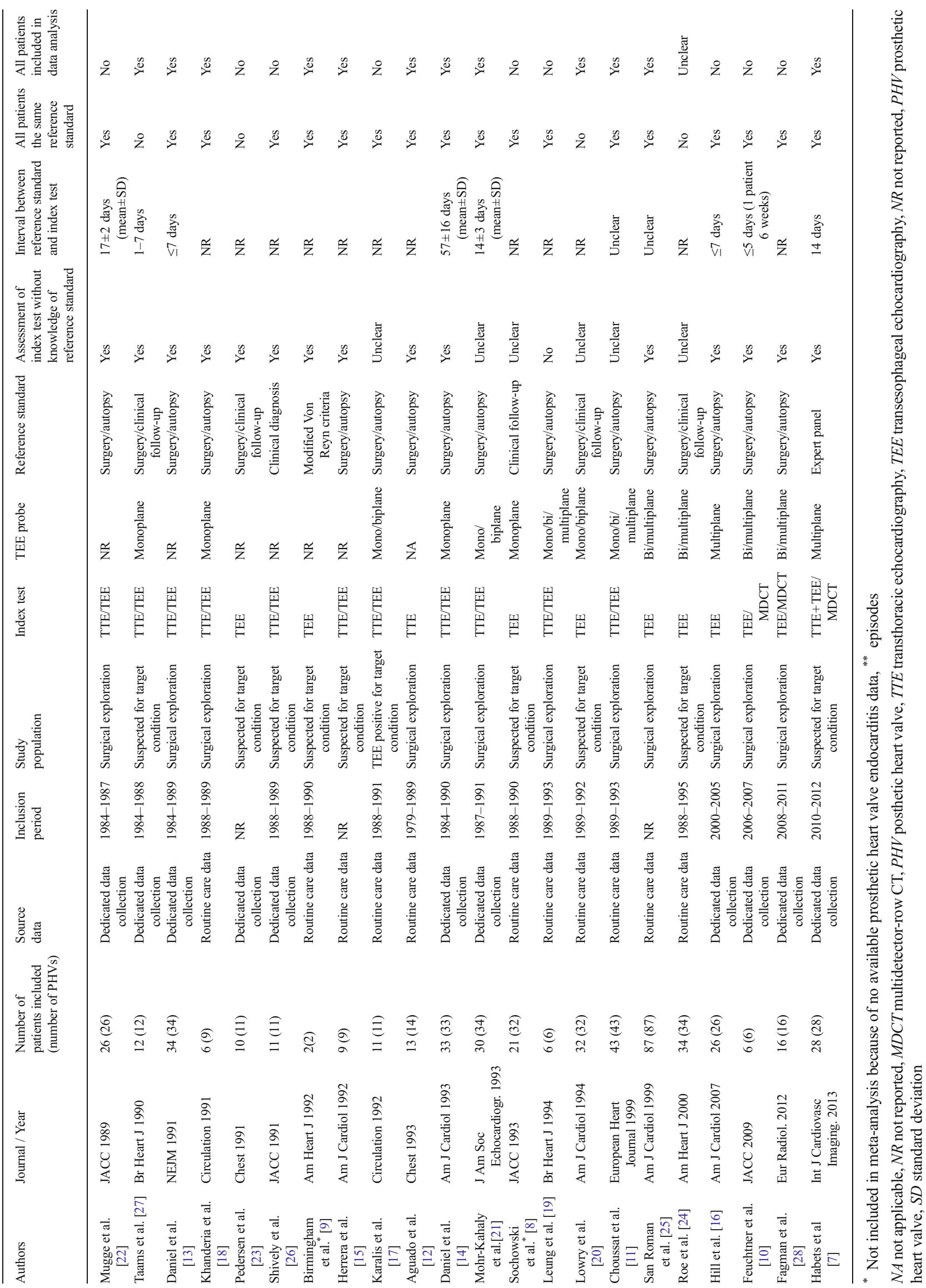


in $14(64 \%)$ studies. In eight studies (36\%), clinical criteria and/or follow-up were mentioned as the reference standard. Less than half of the studies $(n=8,36 \%)$ included multiplanar TEE assessment. The majority of studies $(n=14,64 \%)$ did not report on the interval between the index and reference tests. Two studies had a long time interval ( $>2$ weeks) between index test and reference standard [14, 22]. The other six studies $(27 \%)$ had an acceptable time interval ( $\leq 2$ weeks) [7, 10, 13, 16, 21, 27]. Assessment of the index test was blinded (without knowledge of the reference standard) in $15(68 \%)$ studies. In six of the 22 studies (27\%), it was unclear whether assessment was blinded, and one study included no blinding [19]. The same reference standard was used for all patients in $17(77 \%)$ studies. In $12(54 \%)$ studies, all patients were included in the data analyses. In the meta-analysis, the previously mentioned signs of PHV endocarditis were analysed. Diagnostic accuracy measures for the detection of signs of PHV endocarditis were able to be extracted in 20 studies ( $n=473$ patients) (Table 1$)$.

\section{Vegetations}

Figures 2 and 3 present the sensitivity and specificity of TTE $(n=63)$, TEE $(n=113)$, and MDCT+TEE $(n=50)$ for the detection of vegetations. The pooled TTE sensitivity and specificity for the detection of vegetations were 29\% [95 \% CI: 9-6 $2 \%$ ] and $100 \%$ [95 \% CI: 86-100\%], respectively. TEE (82\% [95 \% CI: 69-90\%]) and MDCT+TEE (88\% [95\% CI: 61-97\%] were more sensitive than TTE (both $p<0.01$ ). No significant difference was found between TEE and TEE+ MDCT $(p=0.60)$, and there were no significant differences in specificity between TTE and TEE/MDCT+TEE (96\% [9 $5 \%$ CI: 81-99\%] and $94 \%$ [95 \% CI: 81-98\%], respectively; $p=$ 0.12 ).

\section{Periannular complications}

The sensitivity and specificity of TTE $(n=172)$, TEE $(n=412)$, and MDCT+TEE $(n=58)$ for the detection of periannular complications are presented in Figs. 2 and 3. The pooled TTE sensitivity and specificity for the detection of periannular complications were $36 \%$ [95 \% CI: 27-46\%] and 93\% [95\% CI: 84-97\%], respectively. One study reported three falsepositive (43\%) TTE examinations [10]. TEE (86\% [95\% CI: $81-90 \%])$ and MDCT+TEE (100\% [95 \% CI: 51$100 \%])$ were more sensitive than TTE $(\mathrm{p} \leq 0.03)$. In one study, TEE failed to detect 5 of 14 (36\%) periannular complications in patients with aortic PHV endocarditis [14]. No significant difference was found between TEE and TEE+MDCT ( $p=$ $0.18)$. There were no significant differences in specificity between TTE and TEE (98\% [95 \% CI: 95-99\%]; $p=0.07$ ) (Fig. 3).

\section{PHV dehiscence}

The pooled sensitivity and specificity of TTE $(n=18)$ for the detection of PHV dehiscence were $11 \%$ [95 \% CI: 1-73\%] and $100 \%$ [95 \% CI: 72-100\%], respectively. No significant difference in sensitivity or specificity was found between TTE and TEE $(n=60)$ (sensitivity of $94 \%$ [95 \% CI: 37-100\%] and specificity of $97 \%$ [95\% CI: 84-99\%]) ( $p \geq 0.05)$. One study reported on the detection of PHV dehiscence with MDCT $(n=16)$ [28]. Fagman et al. [28] reported that seven cases of PHV dehiscence were detected by both TEE and MDCT, although TEE detected three additional cases.

\section{General signs of PHV endocarditis}

The pooled sensitivity and specificity of TTE $(n=55)$ for the detection of general signs of PHV endocarditis were $33 \%$ [95 \% CI: 24-42\%] and $100 \%$ [95 \% CI: 76-100\%], respectively. TEE $(n=114)$ was more sensitive (86\% [95 \% CI: 77$92 \%])$ than TTE $(p<0.001)$. No significant differences in specificity were found between TTE and TEE (95\% [95\% CI: 82-99\%]) $(p=0.29)$. Only one study $(\mathrm{n}=28)$ reported on the detection of general signs of endocarditis by MDCT [7]. In this study, the sensitivity of MDCT+TEE (100\%) was higher than that of TEE (95\%) alone.

\section{Discussion}

We systematically reviewed the literature regarding the use of TTE, TEE, and MDCT for the detection of signs of PHV endocarditis. Notwithstanding the limited number of studies reporting on the diagnostic accuracy of these imaging modalities, non-invasive imaging plays a key role in establishing a diagnosis of PHV endocarditis and has important clinical implications for patient management and outcome. A primary finding in our analysis was that TEE was more sensitive than TTE for the detection of both vegetations and periannular complications, although TEE still failed to detect lifethreatening periannular extensions and vegetations, chiefly in patients with mechanical aortic PHVs (Fig. 4). The addition of retrospectively ECG-gated MDCT to TEE can improve the detection of these periannular extensions and vegetations, primarily in patients with mechanical aortic PHVs. However, limited data are available at the moment, resulting in broad confidence intervals.

According to the QUADAS-2 assessment, several studies were identified as having a risk of bias and/or clinical applicability concerns. This included patient selection (only patients who underwent reoperation), older studies (including monoplanar TEE assessment), unreported blinding for the reference standard, and interval between index test and reference standard (Table 1). 
Fig. 2 Pooled sensitivity analysis of all studies for vegetations and periannular extensions

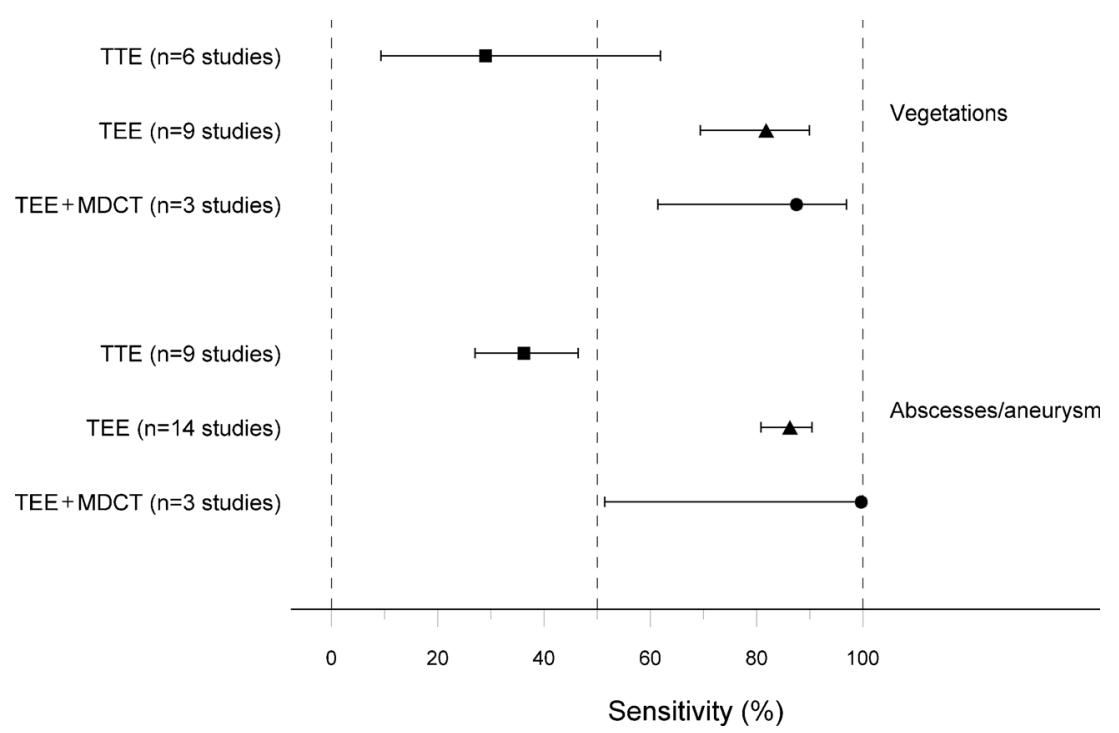

of early mortality are patient age, renal failure, and the presence of an atrioventricular block [11, 13, 16, 25, 30].

The detection of life-threatening abscess formation and mycotic aneurysms with non-invasive imaging is crucial for timely surgical intervention, which can improve the clinical outcome [31]. In this meta-analysis, TEE showed good sensitivity ( $86 \%$ ), and because of its better spatial resolution and the close relationship between the TEE probe and the heart, it was proven to be superior to TTE (36\%) for the detection of abscesses and mycotic aneurysms. Despite this higher level of performance, however, TEE still failed to detect approximately $15 \%$ of the life-threatening abscesses and mycotic aneurysms that were present. These missed periannular extensions were primarily located at the anterior side of the aortic root, which is obscured by acoustic shadowing [13, 16, 21, 27]. In some cases, TTE can offer additional diagnostic value for the detection of anteriorly located abscesses, as assessment of this region is not hampered by acoustic shadowing [27]. In clinical
Fig. 3 Pooled specificity analysis of all studies for vegetations and periannular extensions

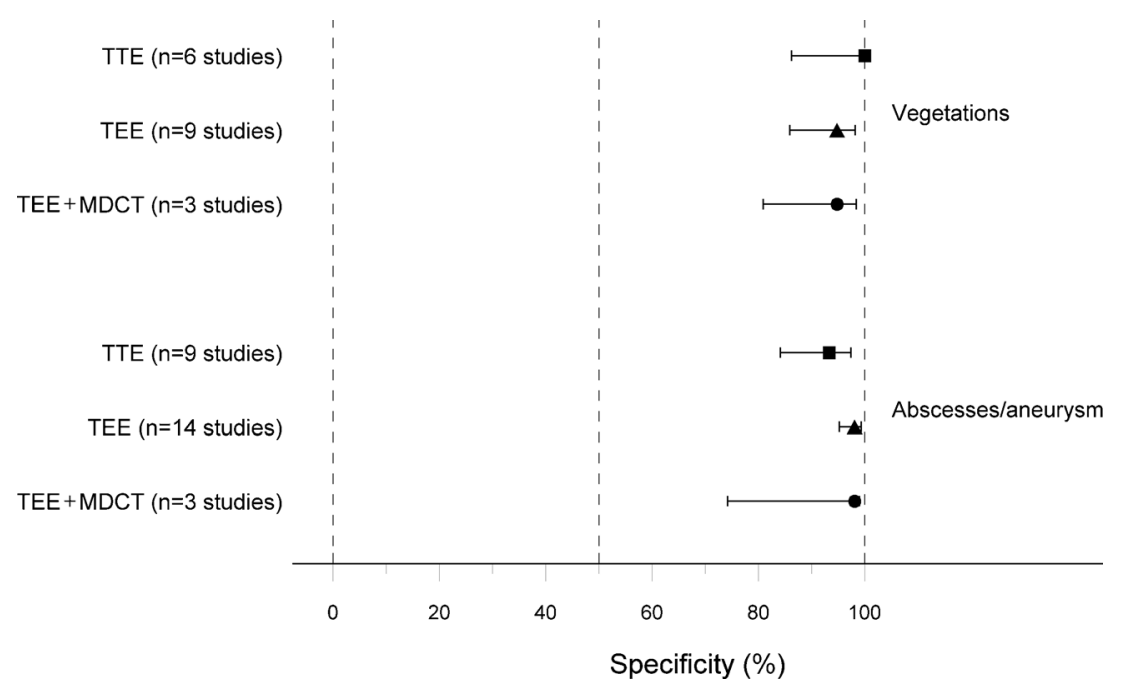


Fig. 4 Complementary value of MDCT+TEE. In this patient with a Carbomedics bileaflet PHV in the aortic position, TTE demonstrated severe aortic regurgitation. In addition, TEE and MDCT revealed a mycotic aortic root aneurysm directly below the right coronary artery (RCA) origin $(\mathbf{a}, \mathbf{c})$, with diastolic paravalvular leakage as seen on colour Doppler imaging (b). While acoustic shadowing on the TEE images (a) hampered complete and accurate assessment of the PHV, MDCT showed no vegetations (c). MDCT nicely demonstrated the close relationship between the mycotic aneurysm and the RCA (c, d). The location of the mycotic aneurysm is indicated by an asterisk on the schematic drawing (d). (Reprinted with permission [35])
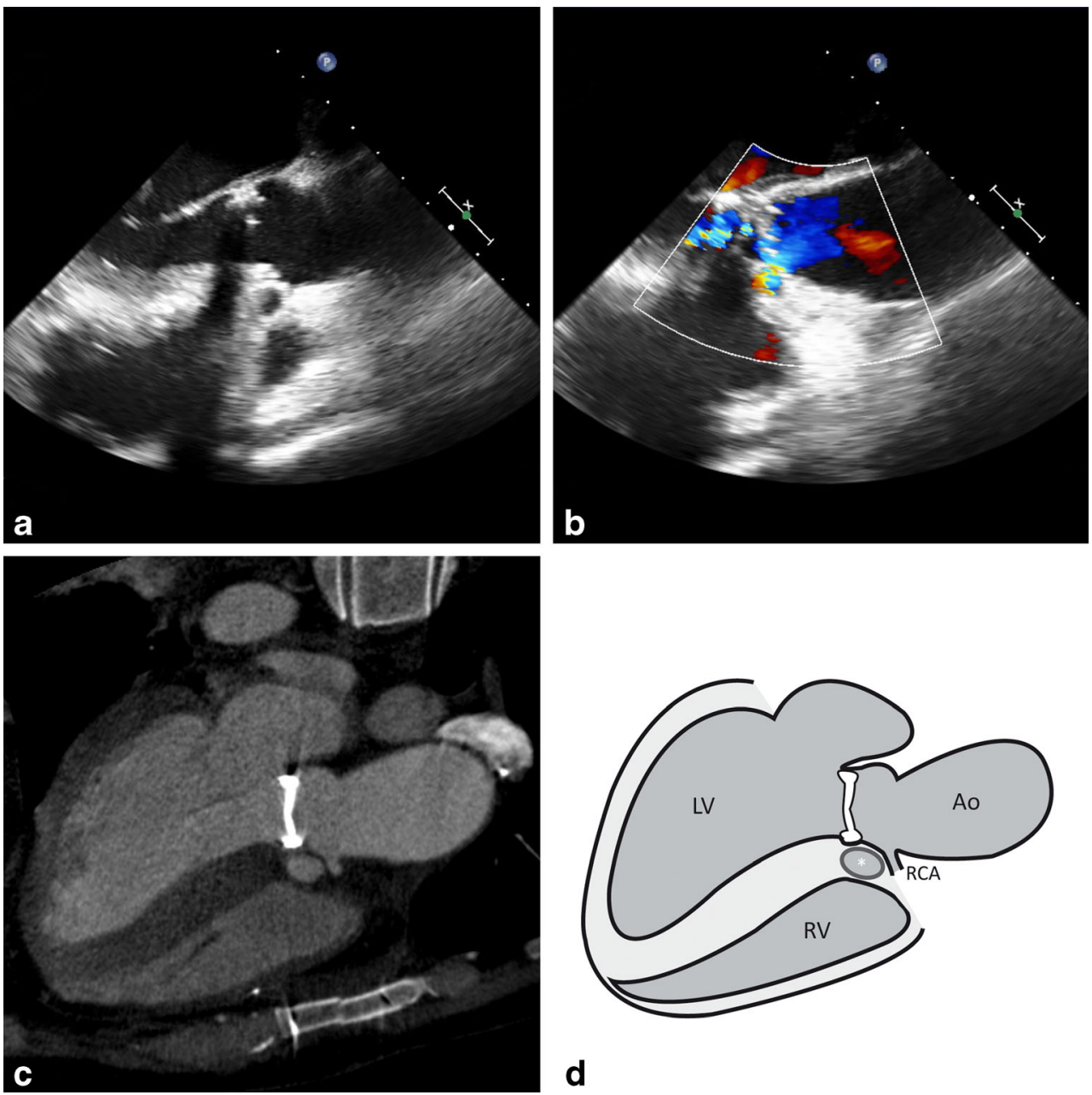

practice, failure to detect periannular complications of PHV endocarditis is unacceptable, and in this context, complementary non-invasive imaging is required. Additional noninvasive imaging may also be valuable for reducing the number of false-positives $(2-7 \%)$ with echocardiography as a means to reduce the risk of unwarranted exposure to a repeat surgery.

This meta-analysis demonstrated that MDCT can provide additional relevant diagnostic information in this high-risk patient group, and should be considered as a complementary imaging technique in routine clinical workup (TTE/TEE), especially in patients with suspected aortic PHV endocarditis. In addition to MDCT, other imaging tools (3D TEE and FDG$\mathrm{PET} / \mathrm{CT}$ ) may also improve the detection of periannular extension. To date, no prospective studies have been published on the diagnostic value of 3D TEE for detection of periannular complications in patients with PHV endocarditis. This technique could be especially valuable in patients with contraindications for MDCT evaluation because of renal impairment. Saby et al. [32] recently prospectively studied the diagnostic accuracy of FDG-PET/CT in PHV endocarditis and assessed the complementary value of PET/CT as a major criterion of the modified Duke criteria in a study of 72 patients with suspected PHV endocarditis. The reference standard was defined as 3 months of clinical follow-up ( $82 \%$ of cases) and/or pathological modified Duke criteria (18\% of cases). The sensitivity and specificity of FDG-PET for PHV endocarditis was $73 \%$ and $80 \%$, respectively. When FDG-PET was added as a new major criterion to the modified Duke criteria, the sensitivity rose from $70 \%$ to $97 \%$. This add-on value of FDG-PET is more or less comparable to the complementary value of MDCT to echocardiography found in our meta-analysis. Moreover, the results of the study by Saby et al. [32] demonstrated that PET/CT missed vegetations in 9/20 (45\%) cases where there were no other signs of PHV endocarditis. The authors mentioned in the discussion that the spatial resolution of FDG-PET/CT was inferior in comparison to TEE. The present meta-analysis shows that the addition of MDCT to TEE may improve sensitivity not only for the detection of periannular extensions, but for vegetations as well. Despite its inferior spatial resolution compared to TEE and MDCT, FDG-PET/CT provides additional metabolic information that can be of value in discerning the presence of active inflammation. However, no reference values exist for FDG uptake 
around PHVs, and therefore specificity is a concern [33]. In addition to signs of PHV endocarditis, FDG-PET-CT can also detect extracardiac infectious foci that may be relevant for patient management (e.g., splenic abscess). Thus, combined contrast-enhanced MDCT and FDG-PET has also been suggested for determining treatment strategy [34]. More prospective studies are needed in order to determine the exact value and position in the diagnostic algorithm of the abovementioned additional diagnostic modalities. PET-CT was not added as imaging modality to our meta-analysis because only one prospective paper [32] was available at the time of the structured search.

\section{Limitations}

This systematic review and meta-analysis had certain limitations. Firstly, in the majority of studies, a selected population was examined - namely, a surgically explored group — and therefore the data provided in this meta-analysis cannot simply be extrapolated to individual patients with suspicion of PHV endocarditis. Furthermore, patients with negative imaging findings will often not undergo reoperation; consequently, this specific patient category was not included in the data analysis in many studies. This paper advocates for more prospective diagnostic cross-sectional studies to determine the exact value of novel non-invasive imaging modalities (3D TEE, MDCT, and FDG-PET/CT) in patients with suspected PHV endocarditis. Secondly, the exact location of missed periannular complications and the sort of TEE probe used (mono/bi/multiplane) was not provided in many of the studies, and so meta-regression could not be performed for these covariates. Thirdly, in a considerable number of the studies, the interval of time between index test and reference standard was either not reported or was too long. This time interval is a critical factor in determining the reliability of diagnostic accuracy measures. In addition, limited MDCT data were available $(n=50)$, resulting in large confidence intervals for the pooled estimates of MDCT+TEE. Prospective MDCT studies are needed to confirm the promising findings of this metaanalysis.

Fourth, in clinical practice, MDCT studies have demonstrated that MDCT can have additional diagnostic value, chiefly in patients with mechanical aortic PHVs. We were not able to perform meta-regression analysis on valve position (aortic vs. mitral position), as this data could not be extracted from the majority of studies and because of the small patient numbers in the limited MDCT data that was available.

\section{Conclusions}

TEE demonstrates good sensitivity and specificity for establishing a diagnosis of PHV endocarditis and for detecting complications associated with high mortality, yet it still misses a substantial number of signs of PHV endocarditis. MDCT in addition to echocardiography may improve diagnostic accuracy in patients with suspected PHV endocarditis, especially in cases of life-threatening periannular extensions requiring urgent reoperation. Further prospective studies are needed to determine the diagnostic value and position in the diagnostic algorithm of additional novel 3D TEE, MDCT, and FDG$\mathrm{PET} / \mathrm{CT}$ imaging modalities.

Acknowledgments We would like to thank Karin A. van Rijnbach, Department of Radiology, University Medical Center Utrecht, for her help with the final editing of the figures. The scientific guarantor of this publication is Prof. Dr. Willem P.Th.M. Mali. The authors of this manuscript declare no relationships with any companies whose products or services may be related to the subject matter of the article. This study was funded in part by a grant from the Dutch Heart Foundation (DHF 2009B014). One of the authors (JR) has significant statistical expertise. Institutional Review Board approval was not required, as the manuscript is a systematic review and meta-analysis. Methodology: diagnostic systematic review and meta-analysis.

Open Access This article is distributed under the terms of the Creative Commons Attribution Noncommercial License which permits any noncommercial use, distribution, and reproduction in any medium, provided the original author(s) and the source are credited.

\section{References}

1. Yacoub MH, Takkenberg JJ (2005) Will heart valve tissue engineering change the world? Nat Clin Pract Cardiovasc Med 2:60-61

2. Habib G, Hoen B, Tornos P, Thuny F, Prendergast B, Vilacosta I et al (2009) Guidelines on the prevention, diagnosis, and treatment of infective endocarditis (new version 2009): the Task Force on the Prevention, Diagnosis, and Treatment of Infective Endocarditis of the European Society of Cardiology (ESC). Endorsed by the European Society of Clinical Microbiology and Infectious Diseases (ESCMID) and the International Society of Chemotherapy (ISC) for Infection and Cancer. Eur Heart J 30:2369-2413

3. Durack DT, Lukes AS, Bright DK (1994) New criteria for diagnosis of infective endocarditis: utilization of specific echocardiographic findings. Duke Endocarditis Service. Am J Med 96:200-209

4. Habets J, Budde RP, Symersky P, van den Brink RB, de Mol BA, Mali WP et al (2011) Diagnostic evaluation of left-sided prosthetic heart valve dysfunction. Nat Rev Cardiol 8:466-478

5. Whiting PF, Rutjes AW, Westwood ME, Mallett S, Deeks JJ, Reitsma JB et al (2011) QUADAS-2 Group. QUADAS-2: a revised tool for the quality assessment of diagnostic accuracy studies. Ann Intern Med 155:529-536

6. Sachdev M, Peterson GE, Jollis JG (2003) Imaging techniques for diagnosis of infective endocarditis. Cardiol Clin 21:185-195

7. Habets J, Tanis W, van Herwerden LA, van den Brink RB, Mali WP, de Mol BA et al (2014) Cardiac computed tomography angiography results in diagnostic and therapeutic change in prosthetic heart valve endocarditis. Int J Cardiovasc Imaging 30:377-387

8. Sochowski RA, Chan KL (1993) Implication of negative results on a monoplane transesophageal echocardiographic study in patients with suspected infective endocarditis. J Am Coll Cardiol 21:216-221 
9. Birmingham GD, Rahko PS, Ballantyne F 3rd (1992) Improved detection of infective endocarditis with transesophageal echocardiography. Am Heart J 123:774-781

10. Feuchtner GM, Stolzmann P, Dichtl W, Schertler T, Bonatti J, Scheffel H et al (2009) Multislice computed tomography in infective endocarditis: comparison with transesophageal echocardiography and intraoperative findings. J Am Coll Cardiol 53:436-444

11. Choussat R, Thomas D, Isnard R, Michel PL, Iung B, Hanania G et al (1999) Perivalvular abscesses associated with endocarditis; clinical features and prognostic factors of overall survival in a series of 233 cases. Perivalvular Abscesses French Multicentre Study. Eur Heart J 20:232-241

12. Aguado JM, Gonzalez-Vilchez F, Martin-Duran R, Arjona R, Vazquez de Prada JA (1993) Perivalvular abscesses associated with endocarditis. Clinical features and diagnostic accuracy of twodimensional echocardiography. Chest 104:88-93

13. Daniel WG, Mugge A, Martin RP, Lindert O, Hausmann D, NonnastDaniel B et al (1991) Improvement in the diagnosis of abscesses associated with endocarditis by transesophageal echocardiography. N Engl J Med 324:795-800

14. Daniel WG, Mugge A, Grote J, Hausmann D, Nikutta P, Laas J et al (1993) Comparison of transthoracic and transesophageal echocardiography for detection of abnormalities of prosthetic and bioprosthetic valves in the mitral and aortic positions. Am J Cardiol 71:210-215

15. Herrera CJ, Chaudhry FA, DeFrino PF, Mehlman DJ, Mulhern KM, O'Rourke RA et al (1992) Value and limitations of transesophageal echocardiography in evaluating prosthetic or bioprosthetic valve dysfunction. Am J Cardiol 69:697-699

16. Hill EE, Herijgers P, Claus P, Vanderschueren S, Peetermans WE, Herregods MC (2007) Abscess in infective endocarditis: the value of transesophageal echocardiography and outcome: a 5-year study. Am Heart J 154:923-928

17. Karalis DG, Bansal RC, Hauck AJ, Ross JJ Jr, Applegate PM, Jutzy KR et al (1992) Transesophageal echocardiographic recognition of subaortic complications in aortic valve endocarditis. Clinical and surgical implications. Circulation 86:353-362

18. Khandheria BK, Seward JB, Oh JK, Freeman WK, Nichols BA, Sinak LJ et al (1991) Value and limitations of transesophageal echocardiography in assessment of mitral valve prostheses. Circulation 83:1956-1968

19. Leung DY, Cranney GB, Hopkins AP, Walsh WF (1994) Role of transoesophageal echocardiography in the diagnosis and management of aortic root abscess. Br Heart J 72:175-181

20. Lowry RW, Zoghbi WA, Baker WB, Wray RA, Quinones MA (1994) Clinical impact of transesophageal echocardiography in the diagnosis and management of infective endocarditis. Am J Cardiol 73:1089-1091

21. Mohr-Kahaly S, Kupferwasser I, Erbel R, Wittlich N, Iversen S, Oelert H et al (1993) Value and limitations of transesophageal echocardiography in the evaluation of aortic prostheses. J Am Soc Echocardiogr 6:12-20

22. Mugge A, Daniel WG, Frank G, Lichtlen PR (1989) Echocardiography in infective endocarditis: reassessment of prognostic implications of vegetation size determined by the transthoracic and the transesophageal approach. J Am Coll Cardiol 14:631-638
23. Pedersen WR, Walker M, Olson JD, Gobel F, Lange HW, Daniel JA et al (1991) Value of transesophageal echocardiography as an adjunct to transthoracic echocardiography in evaluation of native and prosthetic valve endocarditis. Chest 100:351-356

24. Roe MT, Abramson MA, Li J, Heinle SK, Kisslo J, Corey GR et al (2000) Clinical information determines the impact of transesophageal echocardiography on the diagnosis of infective endocarditis by the duke criteria. Am Heart J 139:945-951

25. San Roman JA, Vilacosta I, Sarria C, de la Fuente L, Sanz O, Vega JL et al (1999) Clinical course, microbiologic profile, and diagnosis of periannular complications in prosthetic valve endocarditis. Am J Cardiol 83:1075-1079

26. Shively BK, Gurule FT, Roldan CA, Leggett JH, Schiller NB (1991) Diagnostic value of transesophageal compared with transthoracic echocardiography in infective endocarditis. J Am Coll Cardiol 18: 391-397

27. Taams MA, Gussenhoven EJ, Bos E, de Jaegere P, Roelandt JR, Sutherland GR et al (1990) Enhanced morphological diagnosis in infective endocarditis by transoesophageal echocardiography. $\mathrm{Br}$ Heart J 63:109-113

28. Fagman E, Perrotta S, Bech-Hanssen O, Flinck A, Lamm C, Olaison L et al (2012) ECG-gated computed tomography: a new role for patients with suspected aortic prosthetic valve endocarditis. Eur Radiol 22:2407-2414

29. Tornos MP, Olona M, Permanyer-Miralda G, Almirante B, Evangelista A, Soler-Soler J (1995) Is the clinical spectrum and prognosis of native valve infective endocarditis in non-addicts changing? Eur Heart J 16:1686-1691

30. Graupner C, Vilacosta I, SanRoman J, Ronderos R, Sarria C, Fernandez $\mathrm{C}$ et al (2002) Periannular extension of infective endocarditis. J Am Coll Cardiol 39:1204-1211

31. Habib G, Tribouilloy C, Thuny F, Giorgi R, Brahim A, Amazouz $M$ et al (2005) Prosthetic valve endocarditis: who needs surgery? A multicentre study of 104 cases. Heart 91: 954-959

32. Saby L, Laas O, Habib G, Cammilleri S, Mancini J, Tessonnier L et al (2013) Positron emission tomography/computed tomography for diagnosis of prosthetic valve endocarditis: increased valvular (18)Ffluorodeoxyglucose uptake as a novel major criterion. J Am Coll Cardiol 61:2374-2382

33. Tanis W, Scholtens A, Habets J, van den Brink RB, van Herwerden LA, Chamuleau SA et al (2014) Positron emission tomography/ computed tomography for diagnosis of prosthetic valve endocarditis: increased valvular 18F-fluorodeoxyglucose uptake as a novel major criterion. J Am Coll Cardiol 63:186-187

34. Tanis W, Scholtens A, Habets J, van den Brink RB, van Herwerden LA, Chamuleau SA et al (2013) CT angiography and (1)(8)F-FDG-PET fusion imaging for prosthetic heart valve endocarditis. JACC Cardiovasc Imaging 6:1008-1013

35. Habets J, Tanis W, Mali WP, Chamuleau SA, Budde RP (2012) Imaging of prosthetic heart valve dysfunction: complementary diagnostic value of TEE and MDCT? JACC Cardiovasc Imaging 5:956-961 